\title{
Inhomogeneous Two-Species Annihilation in the Steady State
}

\author{
E. Ben-Naim and S. Redner \\ Center for Polymer Studies and Department of Physics, Boston University, Boston, MA 02215
}

\begin{abstract}
We investigate steady-state geometrical properties of the reaction interface in the two-species annihilation process, $A+B \rightarrow 0$, when a flux $j$ of $A$ and $B$ particles is injected at opposite extremities of a finite domain. By balancing the input flux with the number of reactions, we determine that the width $w$ of the reaction zone scales as $j^{-1 / 3}$ in the large flux limit, and that the concentration in this zone is proportional to $j^{2 / 3}$. This same behavior is deduced from the solution to the reactiondiffusion equation. In the low flux limit, the concentration is almost independent of position and is proportional to $\sqrt{j}$. In the latter case, the local reaction rate reaches maximum at the edges of the system rather than at the midpoint. When the two species approach at a finite velocity, there exists a critical velocity, above which the reactants essentially pass through each other. Results similar to those in one dimension are found in two- and three-dimensional radial geometries. Finally, we apply the quasistatic approximation to our steady-state solution to recover the known time dependence for the reaction zone width for the case of initially separated components with no external input.
\end{abstract}

A fundamental ingredient that controls the kinetics of the two-species annihilation process, $A+B \rightarrow 0$, is the rate of reaction in the interfacial region between a domain of $A$ 's and a domain of $B$ 's. A natural way to study this interface is to prepare a system with initially separated components and then monitor the reaction rate as a function of time, as first studied by Gálfi and Rácz [1]. This type of geometry is also relatively more amenable to experimental investigation [2] than the more extensively studied case of a homogeneous, but random initial condition. In a mean-field description, the kinetics is described by the reaction-diffusion equations

$$
\begin{aligned}
& \frac{\partial c_{A}}{\partial t}=D_{A} \nabla^{2} c_{A}-k c_{A} c_{B}, \\
& \frac{\partial c_{B}}{\partial t}=D_{B} \nabla^{2} c_{B}-k c_{A} c_{B},
\end{aligned}
$$

where $D_{i}$ is the diffusion coefficient of species $i$ and $k$ is the reaction constant. For the case of initially separated components, the initial conditions are $c_{A}(x, t=0)=c_{0}$ for $x>0$ and $c_{A}(x, t=0)=0$ for $x<0$, and conversely for the concentration of $B$ 's. On the basis of scaling arguments, and through solutions of the reaction-diffusion equation, Gálfi and Rácz ${ }^{1}$ found that the width $w$ of the reaction zone increases with time $t$ as $t^{1 / 6}$, and that the reaction rate vanishes as $t^{-2 / 3}$. Numerical simulations appear to confirm this result in two dimensions $[3,4]$ but different exponents appear to hold in one dimension $[5,6]$. Related geometrical properties of the reaction interface have also been studied for the random initial condition [7].

We discuss here new results for the complementary situation in which particles are confined to a finite $d$ dimensional bar with equal fluxes of $A$ 's and $B$ 's injected at opposite ends of the system. Our goal is to understand the geometrical properties of the ensuing steady state. Notice that in the steady state, we can redefine the concentrations by $c_{A} \Rightarrow D_{B} c_{A}$ and $c_{B} \Rightarrow D_{A} c_{B}$ to map the problem to the case of equal diffusion coefficients for the two species. Thus without loss of generality, we posit equal diffusion coefficients for the two species. This system is described by the steady state equations

$$
\begin{aligned}
& D \nabla^{2} c_{A}=k c_{A} c_{B}, \\
& D \nabla^{2} c_{B}=k c_{A} c_{B},
\end{aligned}
$$

with the boundary conditions

$$
\begin{aligned}
\left.D c_{A}^{\prime}\right|_{x=L} & =j,\left.\quad D c_{B}^{\prime}\right|_{x=L}=0, \\
\left.D c_{A}^{\prime}\right|_{x=-L} & =0,\left.\quad D c_{B}^{\prime}\right|_{x=-L}=-j .
\end{aligned}
$$

The reflecting boundary condition imposed on the $A$ 's at $x=-L$, and on the $B$ 's at $x=L$ ensures that the reactants remain within $[-L, L]$. We emphasize that these reaction-diffusion equations provide a mean-field description of the reaction interface in which all spatial fluctuations and the role of the spatial dimensionality is ignored. Within this approximation, we shall determine the extent of the reaction zone and the spatial distribution of the two species.

A rough estimate for these two quantities can be obtained from simple heuristic arguments (Fig. 1). Consider first the situation of a large input flux, so that the concentration profile is linear near the domain boundaries with the magnitude of the slope proportional to $j / D$. If we define the reaction zone as the region for which the concentrations of both species are non-negligible, then the concentration in the reaction zone should be of the order $j w / D$, where $w$ is the reaction zone width. Consequently, the number of annihilation events per unit time is of order $k c_{A} c_{B} w$, which is obtained by integrating the reaction term over the reaction zone. This number should equal the flux of particles $j$ entering the domain. Therefore, balancing these two rates gives

$$
k c_{A} c_{B} w \sim k\left(\frac{w j}{D}\right)^{2} w \sim j
$$

or

$$
w_{0} \sim\left(\frac{D^{2}}{j k}\right)^{1 / 3}
$$


Thus we conclude that the width of the reaction zone is proportional to $j^{-1 / 3}$, and that the typical concentration in the reaction zone is

$$
c_{A}, c_{B} \sim j w / D \sim\left(\frac{j^{2}}{D k}\right)^{1 / 3} .
$$

These results apply as long as the width $w$ is much less than (and independent of) L. From Eq. (5), this corresponds to the flux $j$ being greater than a threshold value $j_{0}$, which scales as $j_{0}=D^{2} / k L^{3}$. When $j<j_{0}$, the rate balance argument fails, indicative of a different scaling behavior for this case. We will outline an alternative approach for this limiting situation based on the approximate solution to the reaction-diffusion equations (see below).

For a quantitative analysis, it is convenient to consider the reaction-diffusion equations for the difference, $c_{-}=c_{A}-c_{B}$, and the sum, $c_{+}=c_{A}+c_{B}$, from which the behavior of $c_{A}$ and $c_{B}$ can easily be inferred. From Eq. (2), $c_{-}$obeys $c_{-}(x)^{\prime \prime}=0$, with the boundary conditions $D c_{-}(x= \pm L)^{\prime}=j$. The corresponding solution is $c_{-}(x)=j\left(x-x_{0}\right) / D$, with $x_{0}$ determined by the constraint that the number of $A$ 's equals the number of $B$ 's in the domain, since the two species are injected and annihilate at the same rate. Thus, imposing $\int_{-L}^{+L} c_{-}(x) d x=0$, gives $x_{0}=0$. This condition also specifies the location of the center of the reaction zone.

Using Eq. (2), the sum of the concentrations obeys the equation $D c_{+}(x)^{\prime \prime}=2 k c_{A} c_{B}$, which can be rewritten in a closed form by using $4 c_{A} c_{B}=c_{+}^{2}-c_{-}^{2}$ to yield,

$$
c_{+}(x)^{\prime \prime}=\frac{k}{2 D}\left[c_{+}^{2}-\left(\frac{j x}{D}\right)^{2}\right],
$$

with the boundary conditions $D c_{+}(x= \pm L)^{\prime}= \pm j$. Since $c_{+}(x)$ is an even function of $x$, it is sufficient to consider $x>0$ only. Notice also that $c_{+} \cong c_{-}$for positive $x$ outside the reaction zone, since the concentration of the minority species $B$ is negligible in this region. (In fact, the ansatz $c_{+}=\left|c_{-}\right|$satisfies the Eq. (7) everywhere, except at the origin.)

For large flux, $j>j_{0}$, we attempt a power-series solution for $c_{+}(x)$ in the reaction zone, namely $c_{+}(x)=$ $c_{0}+c_{2} x^{2}+c_{4} x^{4}+\cdots$. Substituting a three-terms series truncation for $c_{+}(x)$ in Eq. (7), we find

$c_{+}(x)=\frac{w_{0} j}{D}\left[\left(\frac{4}{5}\right)^{1 / 3}+\left(\frac{1}{10}\right)^{2 / 3}\left(\frac{x}{w_{0}}\right)^{2}-\left(\frac{1}{40}\right)\left(\frac{x}{w_{0}}\right)^{4}\right]$

for $|x|<w$, with $w_{0}=\left(D^{2} / j k\right)^{1 / 3}$. In general, the coefficient of $x^{2 n}$ in the series is proportional to $j /\left(D w_{0}^{2 n-1}\right) \sim$ $j^{2(n+1) / 3}$, with the numerical prefactor rapidly converging to a limiting value as more terms in the series approximation fo $c_{+}(x)$ are retained.

We may now determine the reaction zone width by equating $c_{+}$to $c_{-}$at $x=w$. Up to the order given in
Eq. (8), this prescription yields $w \cong 1.179 w_{0}$, in agreement with our previous flux balance argument. From Eq. (8), we also find that the concentration in the reaction zone is of the order of $c_{0} \propto\left(j^{2} / D k\right)^{1 / 3}$. Outside the reaction zone, $c_{+}$matches smoothly with $\left|c_{-}\right|$, as illustrated in Fig. 1. It is worth noting that the above results for $c_{0}$ and $w$ can be inferred by determining the scaled variables that bring Eq. (7) into dimensionless form.

It is instructive to determine the concentration of the minority species outside the reaction zone by direct means. For the concentration of $B$ 's at large $x$, we substitute $c_{A}=c_{B}+(j x / D)$ in Eq. (2) to give $D c_{B}(x)^{\prime \prime}=k c_{B}\left(c_{B}+j x / D\right)$. In the domain $x \gg w$, $c_{B}(x) \ll j x / D$ and therefore the differential equation reduces to the Airy equation,

$$
c_{B}(z)^{\prime \prime}=z c_{B}(z), \quad z=x / w_{0} .
$$

The limiting case $x \gg w$ corresponds to $z \gg 1$, for which the appropriate (decaying) solution is

$$
c_{B}(x) \sim \frac{w_{0} j}{D}\left(\frac{w_{0}}{x}\right)^{1 / 4} \exp \left(-2\left(x / w_{0}\right)^{3 / 2} / 3\right) .
$$

In this expression, the prefactor has been chosen to match the small- $x$ power series representation for $c_{+}(x)$ when $x \cong w_{0}$.

Thus in the high flux limit, the concentration of $B$ 's is approximately $-j x / D$ for $x<-w$ and exponentially small for $x>w$ (Eq. (10)). In the reaction zone itself, $|x|<w$, the concentration of $B$ 's can be deduced by writing $c_{B}$ as $\left(c_{+}-c_{-}\right) / 2$ and using the above expressions for $c_{+}$and $c_{-}$in the reaction zone. This yields $c_{B} \propto j^{2 / 3}$. The concentration of $A$ 's is merely the mirror image of $c_{B}(x)$ about $x_{0}=0$.

In the low flux limit, $j<j_{0}$, the approach presented above fails to satisfy the boundary conditions. However, for this case, we exploit the fact that the concentration must be slowly varying in $x$. Therefore we postulate that $c_{+}(x)=c_{0}+c_{2} x^{2}$ over the entire domain $|x| \leq L$. With this assumption, the boundary conditions on $c_{+}$ at $x= \pm L$ now yields $c_{2}=j / 2 D L$. By substituting this form into Eq. (7), we then find that the constant $c_{0}$ is given by $c_{0}=\sqrt{2 j / k L}$. With these two leading terms, the remaining terms in an infinite power series representation of the solution can be evaluated, and it is straightforward to show that these higher-order terms are negligible. Thus, the approximate form for $c_{+}(x)$ for $j \ll j_{0}$ is

$$
c_{+}(x) \cong \sqrt{\frac{2 j}{k L}}+\left(\frac{j}{2 D L}\right) x^{2},
$$

from which the concentration of each species can be reconstructed by $c_{A, B}=\left(c_{+}(x) \pm j x / D\right) / 2$ (Fig. 2). Notice that the magnitude of the variation in concentration $(\sim j)$ is small compared to the value of the concentration itself $(\sim \sqrt{j})$. More precisely, the ratio $\left(c_{+}(L)-c_{+}(0)\right) / c_{+}(0)$ is proportional to $\left(j / j_{0}\right)^{1 / 2}$. 
A quantity which characterizes the spatial extent of the reaction is the local reaction rate $R(x) \equiv k c_{A}(x) c_{B}(x)=$ $k\left(c_{+}^{2}-c_{-}^{2}\right) / 4$. Using the previously-obtained expressions for $c_{+}$and $c_{-}$, we find

$$
R(x) \propto \begin{cases}(j w)^{2}-\text { const. } \times(j x)^{2}, & j>j_{0} ; \\ j+\text { const. } \times j^{3 / 2} x^{2}, & j<j_{0}\end{cases}
$$

with both constants positive, so that the reaction rate exhibits a unimodal to bimodal transition at $j \cong j_{0}$ (Fig. 3 ). For large flux, the reaction rate is sharply peaked around $x=0$, with a width $w_{0}$, but in the small flux limit, particles are more likely to react near the boundary of the domain rather than in the center.

In the limit of vanishing flux, we can, in principle, find the exact expression for the reaction rate. This limit is defined by the average time between the injection of an $A B$ pair, $t_{j}=1 / j$, being large compared to the average time to diffuse across the domain, $t_{D} \propto L^{2} / D$, so that the system is occupied by at most two particles. To solve for the kinetics of the two-particle system, we define $x_{1}=L-x_{A}$ and $x_{2}=L+x_{B}$, where $x_{A}\left(x_{B}\right)$ is the position of particle $A(B)$. Both variables satisfy $0 \leq x_{1}, x_{2} \leq 2 L$, and a reaction occurs whenever $x_{1}+x_{2}=2 L$ (Fig. 4). By this formulation, the interacting system is mapped onto a two-dimensional random walk in the first-quadrant triangle $x_{1}+x_{2} \leq 2 L$, with reflecting boundary conditions for $x_{1}, x_{2}=0$, and absorbing boundary conditions for $x_{1}+x_{2}=2 L$. To specify the initial conditions, we assume that the input flux at one boundary is uncorrelated with that at the other boundary. Therefore, the first particle attains its asymptotic uniform distribution in the domain before the second particle is injected. For the equivalent twodimensional problem, this leads to the initial condition $p\left(x_{1}, x_{2}, t=0\right)=\left(\delta\left(x_{1}\right)+\delta\left(x_{2}\right)\right) / 4 L$. The reaction rate corresponds to the total flux at the absorbing boundary $x_{1}+x_{2}=2 L$. One way to find this flux is by exact enumeration of the probability distribution for the aforementioned initial distribution of random walks. This method confirms that the reaction rate is indeed bimodal (Fig. 4).

An interesting and natural generalization is the situation where the two species have a superimposed drift toward each other with velocity $v$, as would be the case if the two species where oppositely charged and placed in an external electric field. In the high flux limit, $j>j_{0}$, the density profile exhibits a boundary layer of order $D / v$ from the reaction center, as long as $v$ is greater than $D / L$. Outside the boundary layer the concentration of majority species is $j / v$ and that of the minority species is exponentially small. Inside the boundary layer the drift is negligible and the concentration profile merely reduces to that of the zero drift case in the high flux limit. This approach is valid as long as the boundary layer $D / v$ is larger than the reaction zone width $\left(D^{2} / j k\right)^{1 / 3}$, or $v<v_{0}$, with the critical velocity given by

$$
v_{0}=(D j k)^{1 / 3} \text {. }
$$

In the high velocity limit, we find the amusing feature that the density profile of each species is highly concentrated near the boundary opposite the entrance point. This result can be understood by first rewriting the reaction-convection-diffusion equation in dimensionless form. Introducing $c_{A, B}=\left(v^{2} / D k\right) \tilde{c}_{A, B}$ and $x=D \tilde{x} / v$ yields the scaled equations,

$$
\begin{aligned}
& \tilde{c}_{A}^{\prime \prime}+\tilde{c}_{A}^{\prime}=\tilde{c}_{A} \tilde{c}_{B}, \\
& \tilde{c}_{B}^{\prime \prime}-\tilde{c}_{B}^{\prime}=\tilde{c}_{A} \tilde{c}_{B},
\end{aligned}
$$

with the boundary conditions

$$
\begin{array}{cl}
\tilde{c}_{A}^{\prime}+\left.\tilde{c}_{A}\right|_{\tilde{x}=\tilde{L}}=\epsilon, & \tilde{c}_{B}^{\prime}-\left.\tilde{c}_{B}\right|_{\tilde{x}=\tilde{L}}=0, \\
\tilde{c}_{A}^{\prime}+\left.\tilde{c}_{A}\right|_{\tilde{x}=-\tilde{L}}=0, \quad \tilde{c}_{B}^{\prime}-\left.\tilde{c}_{B}\right|_{\tilde{x}=-\tilde{L}}=-\epsilon .
\end{array}
$$

The scaled flux $\epsilon=\tilde{j}=\left(v_{0} / v\right)^{3}$ is vanishingly small when $v>v_{0}$. To leading order, then, the input is zero, and this implies that the reaction term must vanish, as can be seen by integrating Eq. (14) over the length of the box and using the boundary conditions. This fact suggests a perturbative approach, namely $\tilde{c}_{A}=\tilde{c}_{A 0}+\epsilon \tilde{c}_{A 1}+\cdots$ and $\tilde{c}_{B}=\tilde{c}_{B 0}+\epsilon \tilde{c}_{B 1}+\cdots$. Solving for $\tilde{c}_{A}$ gives $\tilde{c}_{A}=c_{0} \exp (-\tilde{x}-\tilde{L})$ in the vicinity of $-\tilde{L}$ and $\tilde{c}_{A}$ vanishing elsewhere, and conversely for the $B$ 's. Thus the particle are confined to a boundary layer whose width is of order unity in scaled units where $\tilde{L}=L v / D \gg 1$. This zero-order solution can be used in Eq. (14) to obtain the corrections to this leading behavior. Transforming back to the initial variables, we find, to leading order, that the density is proportional to $v^{2} / D k$ within a boundary layer of order $D / v$, and that corrections are of the order $j / v$. This gives a reaction rate which is strongly peaked near the boundaries of the box (Fig. 5). In the low flux limit, we find that this same behavior still applies as long as $v>D / L$.

We can also determine the nature of the reaction zone in a higher-dimensional analog of the finite linear domain. Consider the radially symmetric situation in which the reaction takes place between two concentric hyperspherical shells of different radii, with $A$ 's injected at the outer radius, and $B$ 's injected at the inner radius. The concentration depends on $r$ only, and the corresponding boundary conditions are $D r_{A}^{d-1} c_{A}\left(r_{A}\right)^{\prime}=j, D r_{B}^{d-1} c_{A}\left(r_{B}\right)^{\prime}=0$, and $D r_{B}^{d-1} c_{B}\left(r_{B}\right)^{\prime}=-j, D r_{A}^{d-1} c_{B}\left(r_{A}\right)^{\prime}=0$. The equation for the concentration difference is

$$
\frac{1}{r^{d-1}} \frac{\partial}{\partial r}\left(r^{d-1} \frac{\partial c_{-}(r)}{\partial r}\right)=0,
$$

which, together with the boundary conditions, gives

$c_{-}(r)= \begin{cases}\frac{j}{D} \log \left(r / r_{0}\right) & \text { with } r_{0}=\exp \left(\frac{a^{2} \log a-b^{2} \log b}{a^{2}-b^{2}}-\frac{1}{2}\right) \\ \frac{j}{D}\left(\frac{1}{r_{0}}-\frac{1}{r}\right) & \text { with } r_{0}=\frac{2\left(a^{3}-b^{3}\right)}{3\left(a^{2}-b^{2}\right)} \quad d=3\end{cases}$ 
As in the one dimensional case, the position of the reaction zone center is determined by requiring that $\int_{r_{B}}^{r_{A}} c_{-}(r) r^{d-1} d r=0$. When both radii are large with their difference remaining finite, then $r_{0} \cong(a+b) / 2$. However, if the inner radius is much smaller than the outer radius, then $r_{0} \cong a / \sqrt{e}$ for $d=2$ and $r_{0} \cong 2 a / 3$ for $d=3$. The procedure for finding the individual concentrations of each species closely follows that used for the one dimensional case. In the high flux limit, $c_{+}$is simply equal to $\left|c_{-}\right|$outside the reaction zone, and $c_{+}$ has a power series representation (which is not necessarily even about $r_{0}$ ) within the reaction zone. Following the same method of analysis as in one dimension, we find the same scaling behavior, namely, $w \sim j^{-1 / 3}$ and $c\left(r=r_{0}\right) \sim j^{2 / 3}$. Similarly, in the low-flux limit, we find that the concentration scales as $c \sim \sqrt{j}$, and that the magnitude of the spatial variation of $c$ is of order $j$.

By applying the quasi-static approximation to our description of the steady-state, we can also determine the time dependence of the width of the reaction zone in the Gálfi-Rácz problem, where the two components are initially separated and with no external source of particles. The basis of this approximation is to neglect the time derivative in the diffusion equation and shift this time dependence to a moving boundary condition [8]. For the Gálfi and Rácz problem, the growing depletion layer is the source of the moving boundary which produces the requisite time-dependent flux. For the initial conditions $c_{A}(x, t=0)=c_{0} H(x)$ and $c_{B}(x, t=0)=$ $c_{0} H(-x)$, with $H(x)$ the Heaviside step function, and taking $D_{A}=D_{B}=D$ in Eq. (1), then $c_{-}(x, t)$ satisfies $\dot{c}_{-}=D c_{-}^{\prime \prime}$, with the corresponding initial condition $c_{-}(x, t=0)=c_{0}(2 H(x)-1)$. The solution of this equation is

$$
c_{-}(x)=c_{0} \operatorname{erf}\left(\frac{x}{2 \sqrt{D t}}\right),
$$

with the error function defined by $\operatorname{erf}(y)=$ $\frac{2}{\sqrt{\pi}} \int_{0}^{y} \exp \left(-u^{2}\right) d u$. This solution leads to a depletion zone of the order of $\sqrt{D t}$. The (time-dependent) flux into the reaction zone is thus given by $j(t) \sim D c_{-}(x=0, t)^{\prime} \sim$ $D c_{0} / \sqrt{D t}$. We now use this flux in the steady-state expression for $w$ (Eq. (5)). This yields $w \sim\left(D^{3} t / k^{2} c_{0}^{2}\right)^{1 / 6}$, in agreement with the Gálfi and Rácz result. A posteriori, using the high-flux limit is appropriate, since the flux $j \sim t^{-1 / 2}$ is much greater than $j_{0} \sim L^{-3} \sim t^{-3 / 2}$.

In conclusion, we have investigated the geometrical properties of the reaction zone for two-species annihilation, when particles of each species are injected at a fixed rate $j$ from opposite edges of the system. Our solution to the reaction-diffusion equation provides a mean-field description of a one-dimensional reaction zone geometry. In the limit of large flux, the reaction zone width is proportional to $j^{-1 / 3}$, and the concentration within this zone is of order $j^{2 / 3}$. In the opposite limit, the concentration assumes a nearly constant value of order $j^{1 / 2}$ throughout the system, in which the maximal spatial variation is of order $j$. The former case leads to a sharply localized reaction rate near the domain center, while in the latter case, the reaction rate is maximal near the extremities of the domain. Our approach is easily extended to a variety of potentially relevant situations. Particularly noteworthy is the case of a finite superimposed drift of the reactants towards each other. When the drift velocity exceeds a threshold value, there is a strong tendency for the reactants to pass through each other, a potentially destabilizing mechanism of the reaction interface.

We thank M. J. Stephen for helpful discussions. We also gratefully acknowledge grants from the ARO and NSF for partial support of this research.

\section{REFERENCES}

[1] L. Gálfi and Z. Rácz, Phys. Rev. A 38, 3151 (1988).

[2] Y.-E. L. Koo, L. Li, and R. Kopelman Mol. Liq. Crit. 183, 187 (1990); Y.-E. L. Koo and R. Kopelman, J. Phys. A 65893 (1991).

[3] Z. Jiang and C. Ebner, Phys. Rev. A 42, 7483 (1990).

[4] H. Taitelbaum, S. Havlin, J. E. Kiefer, B. Trus, and G. H. Weiss, J. Stat. Phys. 65, 873 (1991).

[5] S. Cornell, M. Droz, and B. Chopard, Phys. Rev. A 44, 4826 (1991).

[6] M. Araujo, S. Havlin, H. Larralde, and H. E. Stanley, preprint.

[7] F. Leyvraz and S. Redner, Phys. Rev. Lett. 66, 2168 (1991) ; S. Redner and F. Leyvraz, J. Phys. A. 651043 (1991).

[8] S. Redner and D. ben-Avraham, J. Phys. A. 23 L1169 (1990).

\section{FIGURE CAPTIONS}

Fig. 1. Reaction geometry in the steady state. $A$ particles are injected at the right edge and $B$ 's are injected at the left edge, both at the same rate $j$. Shown are the spatial distribution of $A$ 's and $B$ 's (dashed), and the sum of their concentrations $c_{+}(x)$ (solid) in the limit of large flux, $j=100$, based on the numerical solution to the steady-state reaction-diffusion equation for a system with $L=k=D=1$.

Fig. 2. Concentration of $B$ 's in the low flux limit for the case $j=0.01$. The order of magnitude and the variation in this concentration over the extent of the domain are indicated.

Fig. 3. Spatial variation of the local reaction rate $R(x)=k c_{A}(x) c_{B}(x)$ in the case of high flux, $j=100$ 
(solid), and low flux, $j=0.01$ (dashed). The latter data has been multiplied by a factor of 10,000.

Fig. 4. Equivalence between two annihilating random walks in a finite interval of length $2 L$, and a single twodimensional random walk in a triangular domain with reflecting boundary conditions for $x_{1}=0, x_{2}=0$, and absorbing boundary conditions for $x_{1}+x_{2}=2 L$. Also shown is a plot of the flux reaching this boundary, or equivalently, the local reaction rate $R(x)$, as a function of position along the boundary. This data is based on exact enumeration inside a triangle of base 40 after 2000 time steps.

Fig. 5. The density profile in the high velocity limit. Shown are the densities as evaluated by exact enumeration with the following parameters $L=30, v=0.2$, $j=10^{-4}$ and $D=k=1$. 